\title{
Valuing neurosurgery services: Part II. The interdependence of Current Procedural Terminology and federal Medicare payment policy
}

\author{
JAMES R. BEAN, M.D. \\ Neurosurgical Associates, Lexington, Kentucky
}

\begin{abstract}
Current Procedural Terminology (CPT) policies for coding of medical procedures and services are adopted by the American Medical Association CPT editorial panel. Since institution of the Medicare Fee Schedule in 1992, the Medicare budget neutrality rule has strongly influenced CPT policies for the coding of additions or modifications. The Centers for Medicare and Medicaid Services Medicare program policies, particularly payment limits, influence code modification strategies and CPT editorial panel processes.
\end{abstract}

Key Words - Current Procedural Terminology • Relative Value Update Committee • Medicare Fee Schedule - Medicare budget neutrality rule

In Part I of "Valuing neurosurgery services"1 I reviewed the historical development and current interrelationships of CPT and the MFS. In Part II a discussion is provided of how federal influence is structured into CPT policy decisions, even without direct federal control of the CPT program.

\section{INTERDEPENDENCE OF CPT AND RUC PROCESSES}

Until the creation of the MFS and the assignment of RVUs to each CPT code, the reasoning and strategy for creating CPT codes was relatively simple: to have a code that accurately summarized each common procedure with a brief descriptor to allow consistent billing. Often the descriptor was quite general, such as 61510 (craniotomy for supratentorial tumor, nonmeningioma), for procedures that could vary considerably in time and complexity. The general description allowed for simplicity, the assumption being that with one fee for the code at a midlevel complexity, the variation among procedures would average out after a large number of procedures, and the additional effort expended for complex cases would be recouped in the less time and effort for simpler cases.

Traditionally, surgeons charged a global fee for surgical procedures, including operations, hospital visits, and post-

Abbreviations used in this paper: $\mathrm{CMS}=$ Centers for Medicare and Medicaid Services; CPT = Current Procedural Terminology; HCFA = Health Care Financing Administration; MFS = Medicare fee Schedule; RBRVS = Resource-Based Relative Value Scale; RUC $=$ Relative Value Update Committee; $\mathrm{RVU}=$ relative value unit. operative office visits in the lump sum charge. Medicare payments prior to the MFS included such a global payment, and the concept of such a payment was carried over into the design and valuation of the RBRVS. Surveys for procedures included evaluation services just prior to surgery, the number of days in the hospital, and the mean number of postoperative office visits. The initial physician work surveys in the 1980s reflected the customary preoperative day's admission and extended postoperative hospital stays. Only after survey data aquired in the 1980s were enshrined in the RBRVS did a greater number of minimally invasive surgeries, same-day admissions, outpatient surgeries, and early discharges become the norm, under the influence of managed care utilization policies and technical advances. Changes in practice have, for many codes, not been reflected in new RVUs for CPT codes, because the codes have not been subject to new surveys.

Several major policy changes under HCFA rules (the HCFA is now the CMS) changed the assumptions and strategies that underlie CPT code creation and permanently changed the independence of CPT decisions from federal influence through Medicare payment policy. Stated differently, requests for CPT changes and approvals, although nominally controlled by the physician-dominated CPT editorial panel, are often structured to optimize Medicare RVU assignment, or to avoid RVU devaluation.

\section{HOW MEDICARE POLICY INFLUENCES CPT POLICY DECISIONS}

\section{Medicare Budget Neutrality Rule}

First the Medicare budget neutrality rule, which assigns a fixed annual expenditure to each specialty, or at least to 
each category of procedure, is a controlling federal policy that influences CPT coding changes. If codes are revised to expand the number of those describing a given type of service, such as craniotomy for aneurysm, MFS valuation takes into account the total annual expenditure by Medicare on that type of procedure in prior years, and for every increase in payment level for new more complex codes it assigns a decreased value to older, simpler codes, so that the total Medicare expense for that type of procedure remains unchanged.

Thus, when a request was made in 1999 to create a code involving craniotomy for complex carotid artery circulation aneurysm (61697) in addition to the standard code (61700), it was recognized that the complex code would draw value from the standard code, devaluing the "noncomplex" aneurysm code (61700). Because the actual frequency of "complex" compared with "simple" aneurysm cases was unknown, a strategic decision was made to request a "tracking" (Category III [emerging technology]) CPT code, which is used to determine the frequency of use. Thereafter, two pieces of information will be needed to assign a new value: first, the amount of additional work involved in the average "complex" aneurysm, as determined by survey data; second, the percentage of cases coded and billed to Medicare as "complex" (61697). An accurate calculation of the new code can then be assigned without excessively devaluing the original code. For instance, if complex aneurysms are coded $20 \%$ of the time and are found on surveys to be a mean of $30 \%$ greater work, that additional work of $30 \%$ will be extracted from the remaining $80 \%$ of simple aneurysm procedures coded (61700). Because the volume of 61700 codes would be fourfold (80:20 ratio) the number of complex codes (61697), 61700 would be reduced in work value by $30 \%$ / $4=7.5 \%$. Currently in the 2002 MFS, both 61697 and 61700 are paid at the same rate.

Although ideally a code is created only to clarify the procedure performed-with a unique summarizing descriptor, and a more elaborate vignette to describe a typical case in some detail-it is no longer created in isolation from the RBRVS value it will acquire or the effect it will have on other code values. No code is independent; each has an effect on the value of codes within its family and on all other codes in the CPT compendium. New codes assigned for older procedures performed using new techniques borrow funding from the pool for the older codes. New technology that does not duplicate prior techniques is designated as a new category of service in which there is allocation of funding that does not diminish a particular pool. Medicare rules, however, preserve budget neutrality by decreasing the subsequent year's conversion factor to accommodate any new technology expense exceeding $\$ 20$ million in projected Medicare spending. Further, because greater than $80 \%$ of private payers now base their fees on the MFS, with their own unique modifier or as a percentage of the Medicare fee, Medicare payment policies permeate the entire public and private health care sector.

\section{Risk of Reimbursement Reduction}

Second, each time a newly created CPT code is more than an editorial change- that is, one that creates a code with a new value- - the new code is sent from the CPT editorial panel to the RUC for valuation. The RUC valuation provides organized medicine the opportunity to find the proper niche for the procedure in the RBRVS labyrinth, rather than relying on government agency determination alone. The value determination made by the RUC is only a recommendation to the CMS, which can be accepted, rejected, or modified, although it is usually determinate of the final value. The valuation process involves notifying specialty societies of the new code and determining their level of interest - that is, whether there is 1) no interest, 2) interest in commenting on proposed values, or 3) interest in surveying the specialty society's membership to generate a proposed relative value. The distributed survey asks about operative duration, surgical intensity, and the number and complexity of preservice and postservice visits. The requesting specialty presents the survey data to the RUC, which is composed of a broad array of other specialties, that, if the requesting specialty is successful in its request (budget neutrality rule), stand to lose value through a reduced annual conversion factor. Finally, a work RVU value is approved by the RUC and forwarded as a recommendation to the CMS.

If the request is a revision to an older-style procedure, such as endoscopic codes rather than open surgery codes for intraventricular operations, the new valuation may produce the following: 1) reexamination and revaluation of the older (reference) codes, with possible devaluation based on changes in current practice, such as fewer hospital visits; and 2) values considerably less than those associated with the older open procedure codes, because of shorter operative times, fewer hospital days, and fewer office follow-up visits (thus reimbursed at a lesser value than when the older codes were used). Either way, reimbursement decreases. In this case, budget neutrality does not apply, and the savings accrue to Medicare.

\section{Bundled Compared With Itemized Coding}

Third, bundled surgical services have been a fundamental part of the RBRVS design. Medicare procedural fee reductions, however, have occurred successively over the past 10 years: first as work relative values; then as practice expense relative values were each implemented over sequential 4-year periods from 1992 to 1996, and 1999 to 2002. Individual reductions in code fees have been paralleled by an increase in the number of codes added to the base code to capture compensation for additional work. The traditional strategy of a simple bundled surgical code that absorbed variations in case-related complexity and additional surgical techniques, which was adjusted over time by increases in a practice's charge and payment received for the code, has given way to a strategy of increased "granularity," or itemization of the components of the service. When global fees could no longer be adjusted to accommodate growing procedural complexity, "unbundled," or add-on codes and modifiers became the substitute strategy.

Nowhere has this tendency to unbundle, or itemize, been more evident than in complex spinal surgery, in which a spinal decompression and fusion may have six or more codes, such as 63047 (decompressive laminectomy), 63048 (additional-level laminectomy), 22842 (pedi- 
cle screw stabilization), 22630 (posterior lumbar interbody fusion), 22632 (additional level interbody fusion), 22851 (application of intervertebral biomechanical device), 20937 (iliac crest morselized bone graft), and perhaps even 22612 (posterolateral fusion). As additional components are added to the procedure, they are explicitly itemized and included as an additional charge, because the base global fee is fixed by regulation. Although physicians cannot alter global fees within the MFS, they can increase the value by itemizing new components of the procedure as add-on codes.

The HCFA's response to perceived unbundling of claims was to contract for development of computer software that can detect CPT code-based claims, which should not be submitted simultaneously, either because one code duplicates the service included in another or because the two services are not or cannot be performed simultaneously. The program, termed the Medicare Correct Coding Initiative, performs automatic computer "edits" of submitted claims, rejecting those with codes listed as incompatible in the software. Initially the edits were confidential, giving rise to the term "black box edits," because HCFA aimed to prevent "gaming" or physician circumvention of the edit system. The claim rejections, however, were disruptive to the submission of medical office claims-rejections required repeated submissions and some coding conflicts could not be understood by physician office administrators at all. Subsequently, Correct Coding Initiative coding edits have been made available to the public (at a price), and new edits are annually submitted to participating CPT societies for comment prior to implementation.

\section{Current Procedural Terminology Coding to Capture Office Overhead Expense}

Fourth, because payment for physician office expenses is now determined explicitly through a resource-based practice expense component of the MFS, codes have been introduced that describe no physician work and contain no "physician work" values, but rather describe work performed by allied health personnel or the use of office equipment, supplies, or devices that cost the practice money in overhead expense. Because the payment for physician overhead expenses cannot be recouped from Medicare except through CPT codes, which prior to 1992 were designed for work performed by physicians with a chargebased overhead expense averaging approximately 50\%, the necessity for practice expense-only CPT codes is a direct offspring of Medicare payment policy. One such code considered at the November 2001 CPT Editorial Panel meeting was for refill of an intrathecal catheter opioid pump reservoir. Preliminary evidence indicated that refilling was often, perhaps $50 \%$ of the time, performed by clinical nursing personnel, without direct involvement of the physician; although the activity was physician directed, the expense incurred by the practice was created by the nurse time and supplies used.

\section{Confidentiality of CPT Proceedings}

Fifth, the CPT editorial process traditionally was closed to the public. This format was designed to permit free and uninhibited discussion, like a jury able to hear testimony and evidence, that would be unbiased by the presence and influence of special interests during deliberations. Because CPT coding policy decisions have effectively become federal payment policy, however, the demand to open up meetings has grown. Specialty advisors have been allowed to observe the deliberations, although voting remains by secret ballot and results are not revealed until incorporated into written policy.

Recently, the demand that the CPT process conform to public policy openness has become more pressing. The Health Insurance Portability and Accountability Act of 1996 requires national code sets and classification systems, and regulation requires public openness in setting policy. Because CPT is designated by the CMS as the medical coding standard, the CPT process will have to conform to federal policy-setting standards if it is to retain its unique designation. Additionally, the National Committee on Vital and Health Statistics has expressed concern that the CPT process lacks openness, reserving the option of recommending an alternative coding system in the future, if satisfactory public openness is not achieved. Further, although the CPT editorial panel is not a government body, it strongly influences federal policy, by adoption of CPT policies within Medicare, and thus may be considered bound by the Federal Advisory Committee Act, which requires the following: 1) advance public notice of meetings; 2) public meetings and opportunity for public comments; 3) public documentation of meetings; and 4) public access to records (McCann, Bart: Expansion of the CPT Editorial Process, Health Policy Alternatives, Washington, D.C. Presented at the November 2001 CPT/ Health Care Professionals Advisory Committee Meeting, San Diego, CA). The editorial panel may have to make the aforementioned adjustments to fulfill the public policy role it has chosen and, as such, may significantly diminish the hold panel members and medical specialty societies have on coding policies.

One potential byproduct of CPT Editorial Panel openness to public comment is the growing influence that medical product vendors have on CPT coding policy. Medical device and equipment manufacturers have discovered that their products are more likely to be used by physicians if a separate CPT code for the product exists, allowing additional reimbursement to the physician. Device manufacturers are increasingly requesting new codes for their products from the CPT panel, either directly or indirectly through medical specialty societies. The request is judged based on professional assessment of the device's proven effectiveness, frequency of use in medical practice, and Food and Drug Administration approval. With increased public openness regarding the CPT process, code approval is more likely to be influenced by commercial marketing tactics rather than solely by the panel members' professional judgment of medical effectiveness.

\section{Evaluation and Management Documentation: Clinical Relevance Compared With Medicare Accounting}

Finally, evaluation and management codes were added to the CPT compendium in 1992, coincident with the MFS, to accommodate Medicare's need for nonprocedural service codes. Over $40 \%$ of physician payments through the MFS are for evaluation and management services, which amounted to over $\$ 17$ billion in Medicare payments 
in 2001. Codes were established for differing levels of complexity, with higher RVUs assigned for more complex levels of service. Although these codes and descriptors were created by CPT, the HCFA wrote Medicare evaluation/management documentation guidelines in 1995, explaining the amount of detail required in medical reports to qualify for a given level of service and payment. The controversy over CPT evaluation/management coding instructions and Medicare evaluation/management documentation guidelines has made explicit the interdependency but often conflicting perspectives of physician service coding and Medicare payment policy.

Because the MFS had the explicit purpose and design to increase the value of general medicine evaluation and management (nonprocedural) services, the guidelines were written to illustrate a typical general medical evaluation. Specialties, neurosurgery included, were disadvantaged by the guidelines because higher complexity codes required broad and detailed multisystem evaluations, whereas specialty evaluations, by their very nature, focused in great detail on one or at most a few systems. The guidelines were amended several times (in 1997, 1999, and 2000) in response to physician complaints. Each revision allowed more value for specialty examinations, but retained the general history format and became more prescriptive of the precise number of elements to be used in the evaluation/management reports. Because each iteration emphasized items that could be counted by reviewers or auditors, incentives were created to load the medical report with detail irrelevant to the understanding the medical problem and unnecessary to its treatment.

After the Balanced Budget Act of 1997, with its emphasis on creating budget savings in Medicare by reducing fraud and abuse, evaluation/management coding became the growing focus of federal fraud and abuse investigations against physicians. Attempts to resolve the conflict involving Medicare's use of the evaluation/management service medical report as a document with which to audit the billing rather than as an accurate communication about the medical condition and treatment have failed. In 2002 the issue was brought before a CPT Workgroup for development of new recommendations. This conflict exemplifies how the CPT panel cannot make rules in isolation from federal policy implications, and the confusion and disagreement over evaluation/management documenta- tion illustrate graphically the chaos created when either CPT or CMS members make coding policies without soliciting consensus agreement.

\section{CONCLUSIONS}

Price is a negotiated agreement over value, whether set at a corporate level based on supply and demand, agreed upon by individuals at the point of purchase, or established as statutory public policy. Medical services are indirectly priced (that is, made price eligible) by organized medicine through the CPT editorial panel after being accepted into the CPT compendium and receiving an individual code, and thus billing status. Medical services are directly priced according to RVUs recommended by organized medicine through the RUC and finally determined by the CMS in the annual MFS. The negotiation over value within organized medicine and between organized medicine and the CMS occurs both at the RUC, where value becomes a numeric quantity, and in the CPT editorial panel, where services are recognized as legitimate and compensable, warranting recognition and separate value.

The interrelationship between CPT and the RUC has grown so intimate that coding policy is often a direct reaction to federal Medicare payment policy, not simply a response to medical practice, physician convenience, billing simplicity, or private billing needs. The 1992 MFS permanently linked physician payment and CPT coding rules to federal Medicare policy and budgetary limitations, the limitations of which have grown progressively more stringent over the past decade.

\section{References}

1. Bean JR: Valuing neurosurgery services: Part I. The historical development and interrelationships of Current Procedural Terminology and the Medicare Fee Schedule. Neurosurg Focus 12 (4):Article 1, 2002

Manuscript received December 19, 2001.

Accepted in final form March 7, 2002.

Address reprint requests to: James R. Bean, M.D., Neurosurgical Associates, 1401 Harrodsburg Road, Suite B485, Lexington, Kentucky 40504. email: jbeanlex@aol.com. 